\title{
Study of MicroRheology Characterization on Soft Microgel
}

\author{
Yinzhu Ye $\mathrm{e}^{1,2, \mathrm{a}}$, Xingcai $\mathrm{Wu}^{1,2, b}$, Yuwei He $\mathrm{He}^{3, \mathrm{c}}$ \\ ${ }^{1}$ Research Institute of Petroleum Exploration \& Development, PetroChina, 10083, China, \\ ${ }^{2}$ Key Laboratory of Oil \& Gas production, CNPC, ${ }^{3}$ Beijing LDS Technology CO., LTD, 100101, China \\ aemail: yeyinzhu@petrochina.com.cn, bemail:wuxingcai@petrochina.com.cn, \\ cemail:ellen.he@Idschina.com.cn
}

\begin{abstract}
Keywords: Soft Microgel, Improved oil recovery, MicroRheology Characterization, In-deep conformance control
\end{abstract}

\begin{abstract}
A new particle-type polymer SMG is developed in high temperature and high salinity reservoirs. This technology is one kind of deep profile control techniques which can remarkably improve oil recovery in the oilfield. Rheometry is the main characterization method of chemical agents in deep profile domain, such as SMG. Because SMG is dispersion system and the viscosity value and elasticity value of SMG is very low, it is very difficult to measure the viscosity of SMG using traditional Rheology method. The MicroRhemetry has the advantages of non-destructive measurement of original state, accurate, convenient and reliable results etc.. And also it is a supplementary means of study on microscopic displacement mechanism. These results could have some reference meaning and guiding significance for other similar gel system.
\end{abstract}

\section{Introduction}

A new particle-type polymer does not have a long chain structure, but have a spatial network. It is microsphere as microscopic appearance. Because it can absorb water and expand, and has deformation ability, it is named Soft Microgel (SMG). It obtains great improvement in temperature and salinity resistance [1] and can be counted on to solve the need of EOR for high temperature and high salinity reservoirs. It is a type of dispersion in water when working, and can dynamically modify the microscopic permeability to achieve high efficient modification of water to oil mobility ratio [2]. The SMG can be easily stored and have good effect on profile control in reservoirs [3].

In this paper, first, the Mechanism of MicroRheology is illustrated. Then the experiment process is designed and laboratory experiment is carried by using MicroRheology method.

\section{MicroRheometer Principle}

Multispeckle Diffusing Wave Spectroscopy is used in lab analysis of soft materials. The heart of the MicroRheometer is a detection head which moves up and down along a flat-bottomed cylindrical cell. The light source is an electro luminescent diode in the near infrared (880nm). Two synchronous optical sensors receive respectively light transmitted through the sample $\left(180^{\circ}\right.$ from the incident light, transmission sensor), and light backscattered by the sample ( $45^{\circ}$ from the incident radiation, backscattering detector). The signal is first treated by controller of MicroRheometer [4] [5]. Elasticity factor, viscosity factor, elasticity modulus and viscous modulus are very important parameters of MicroRheology. The physical meaning of elasticity modulus and viscous modulus is the same as that of traditional Rheology.

Mean Square Displacement(MSD). MSD is used to quantify the inner particle movement of the sample. The value is the average of a large number of scattering trajectory where the unit is $\mathrm{nm}$. The curves of MSD versus ageing time are named MSD Curves or Decorrelation Curves. The MSD curves are the signature of the rheology of the sample. They report the visco-elastic behavior of the sample. By acquiring MSD curves at several ageing times, the evolution of both elastic and viscous properties of the sample i identified.

Elastic factor(EF). The elasticity factor is directly equal to the inverse of the MSD plateau level. 
It is calculated for each MSD to see its evolution versus ageing time of the sample.

$$
E F=\frac{1}{6 \delta^{2}}
$$

In the type: $6 \delta^{2}$ is MSD plateau level, $\mathrm{nm}^{2} ; E F$ is elastic factor, $\mathrm{nm}^{-2}$

Viscosity factor(VF). The viscosity factor evolves with the evolution of the MSD curves at long decorrelation time, it corresponds to the displacement of the curve to the right and reflects the viscosity evolution in the product.

$$
V F=\frac{1}{6 D_{m}}
$$

In the type: $D_{m}$ stands for diffusion coefficient, $\mathrm{nm}^{2} \mathrm{~s}^{-1} ; V F$ stands for viscosity factor, $\mathrm{nm}^{-}$ ${ }^{2} \mathrm{~S}$

Visco-elastic moduli(G' G"). The evolution of the visco-elastic moduli versus time at one frequency allows to follow the evolution of the elasticity and viscosity of the product after sampling. It gives a useful information on the time of restructuration when the moduli reach a plateau. From one MSD curve (at one ageing time) can be calculated the visco-elastic moduli versus frequency (inverse of decorrelation time).

$$
\tilde{G}=\frac{K_{b} T}{\pi a S\left\langle\Delta \tilde{r}^{2}(s)\right\rangle}
$$

In the type: $\tilde{G}$ stands for shear moduli, Pa; $K_{b}$ stands for Boltzmann's Constant, J.K $\mathrm{K}^{-1} ; a$ stands for particle mean diameter (d50), $\mu \mathrm{m} ; S$ is Laplace frequency; $\Delta \tilde{r}^{2}(s)$ is Laplace transformation of MSD

\section{Experimental}

Materials. SMG is made of monomers of polymer, crosslinker agents and some assistants such as white oil by means of inverse emulsion polymerization. It has been applied in industrial production in some Oil Reservoirs in China, for example Huabei Oilfield and Dagang Oilfield. The particle effective concentration of SMG(micrometer-scale) system in dispersion is $1 \%$ by using deionized water and swelling for 15 days.

Experimental Apparatus. In this experiment the MicroRheometer is used which is named RHEOLASER ${ }^{\circledR} L A B$ of Formulaction Company in France.

Experimental procedure. (1)sample bottle is washed clean and dried; (2)Sample of SMG mixing uniformity is poured into the sample bottle $(20 \mathrm{ml})$ slowly; (3) The bottle is put into the apparatus; (4) Relevant parameters is imported in the software in the computer, and start key is clicked. The temperature is $26^{\circ} \mathrm{C}$ and it takes 7 days in the experiment.

\section{Results and Discussion}

Raw data (the MSD curves) are showed in Figure 1. They are the signature of the rheology of the sample. The visco-elastic behaviour is reported in the sample. By acquiring MSD curves at several ageing times, we can identify the evolution of both elastic and viscous properties of the sample. It is observed that the acquisition curves move from short to long decorrelation time (from left to right) due to the structure recovery of the samples after the sampling. The viscosity and the elasticity increase versus the ageing time. 

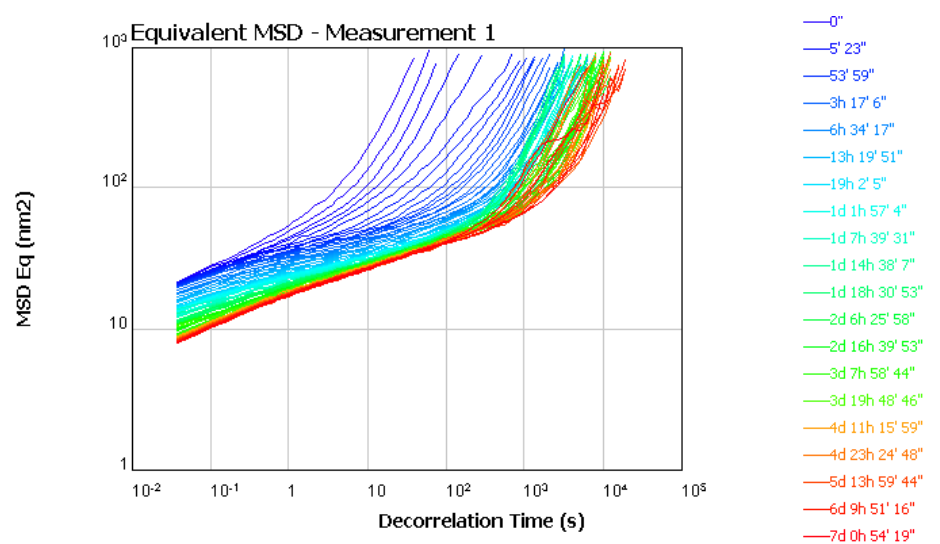

Fig.1. Mean Square Displacement

The elasticity increases after sampling, it is sensitive to the shear applied during sampling. The sample recovers $80 \%$ of its elasticity 2 days after sampling, as shown in Figure 2 . It is given in Figure 3 that the elastic modulus $G^{\prime}$ at $1 \mathrm{~Hz}$ provides the same information as the elasticity factor. The elasticity increases after sampling due to restructuration. The elastic modulus G' recovers $80 \%$ of its structure after 1 day 4 hours.
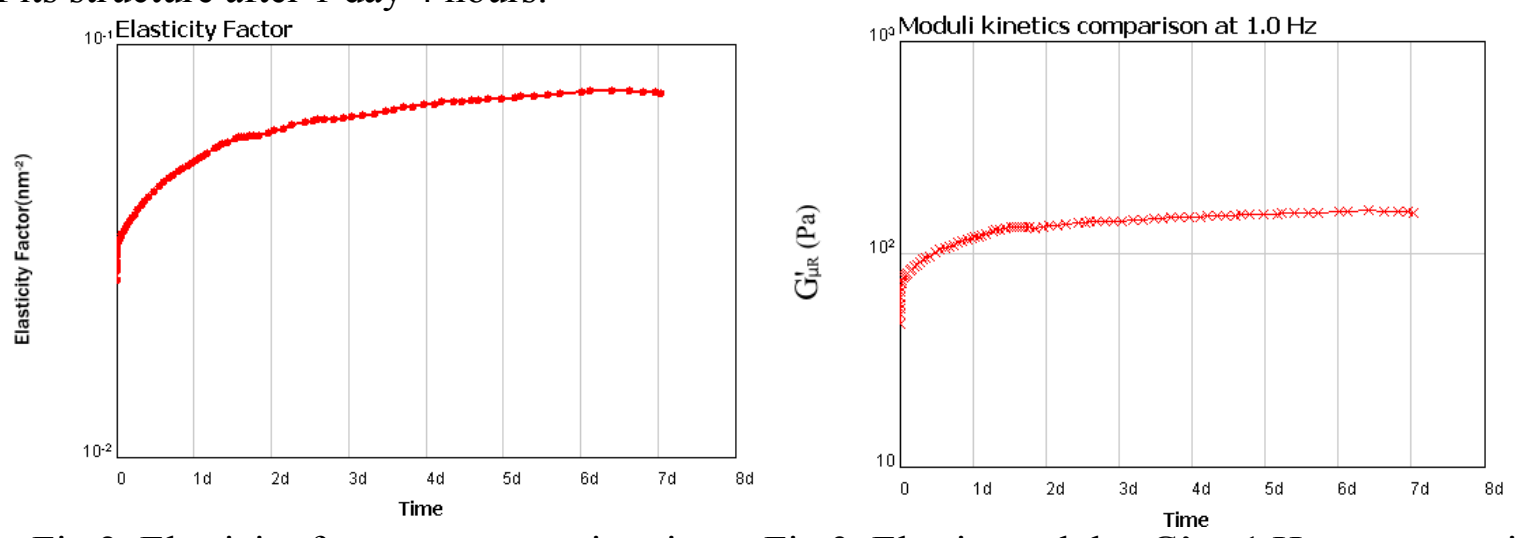

Fig.2. Elasticity factor versus ageing time

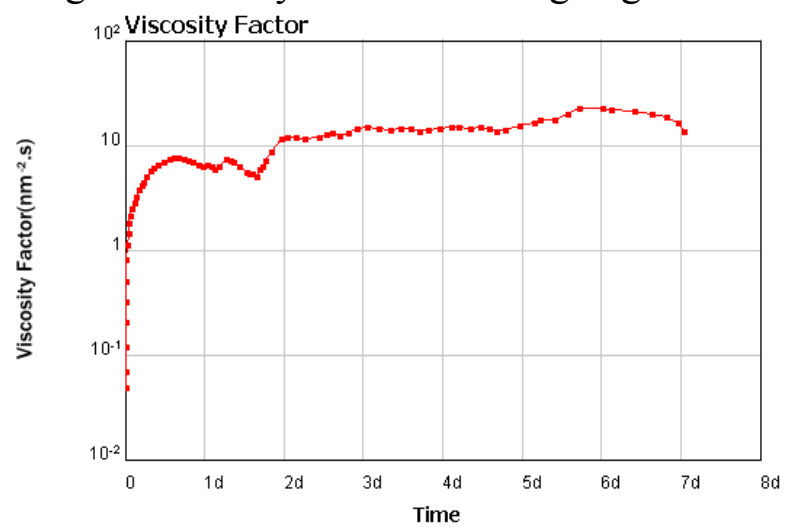

Fig.4. Viscosity factor versus ageing time

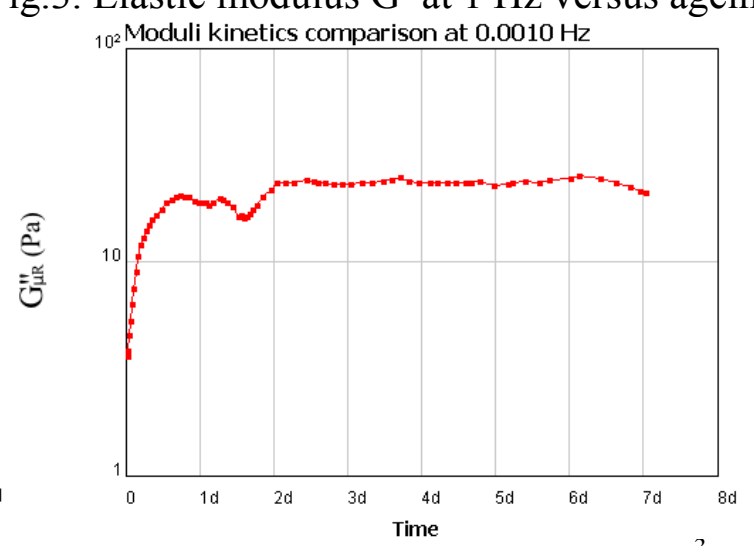

Fig.5. Viscous modulus G" at $10^{-3} \mathrm{~Hz}$

It is shown in Figure 4 that the viscosity increases after sampling due to the restructuration after sampling. The wavering between 0 and 2 days are due to the thermal variations. The viscosity reaches a complete equilibrium after 5 days 17 hours. It starts to decrease after 6 days $1 / 2$ meaning a beginning of destabilisation, and the sample starts to become unstable. Figure 5 gives the modulus versus ageing time for the sample at $10^{-3} \mathrm{~Hz}$. It is noticed that the values of G', reach a plateau after 2 days and start to decreases after 6 days $1 / 2$, meaning a beginning of destabilisation.

Rheolaser LAB allows to calculate the visco-elastic moduli versus frequency from the MSD curve, just by inputting the particle mean diameter. The figure 10 gives the moduli at different ageing times after sampling. The moduli are measured at rest, it is a non intrusive method. The spectra shows that the sample is visco-elastic with a typical behavior: a dominant elastic behavior between $10^{-3}$ and $10 \mathrm{~Hz}$, and a dominant viscous behavior at very low frequency (under $10^{-3} \mathrm{~Hz}$ ), as shown in Figure 6. 


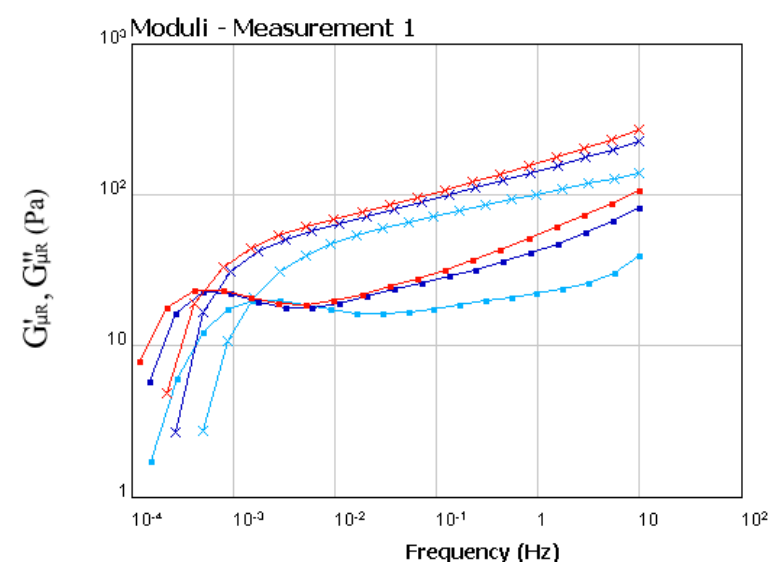

Fig.6 Visco-elastic moduli G' G' versus frequency

\section{Conclusion}

According to this paper, the main conclusions concerned are as follows:

1. The MicroRheology method has the advantages of non-destructive measurement of original state, convenient, accurate and reliable results etc..

2. The sample has a dominant viscous behavior under $10^{-3} \mathrm{~Hz}$; The sample has a dominant elastic behavior over a large frequency range: between $10^{-3} \mathrm{~Hz}$ and $10 \mathrm{~Hz}$ (4 decades).

3. Some problems need further research according to influence degree of visco-elasticity versus EOR/IOR and some research work is needed to do. Some chemical agents, for example, Partial Hydrolyzed Polyacrylamide is very difficult to study, so some inorganic particles which have special suspension are put into water dispersion. And the Brownian movement of the inorganic can be measure to characterize the micro visco-elasticity of chemical agent.

\section{Acknowledgment}

This study is supported by the National Major Science and Technology Special Project: High-efficient in-depth fluid diverting and fine layered injection-production technology (2011ZX05010-003), and also the author would like to thank the support of Beijing LDS Technology CO., LTD.

\section{Reference}

[1] Xingcai Wu, Chunming Xiong, Hanbing Xu, etc,: A Novel Particle-Type Polymer and IOR/EOR Property Evaluation, SPE-177421,Abu Dhabi International Petroleum Exhibition \& Conference, Abu Dhabi, UAE, 9-12 November, 2015

[2] Yinzhu Ye, Zhengbo Wang: Study on Percolation Behavior of New Soft Movable Micro-Gel, Xin Jiang Petroleum Geology Vol.32 No.2, 2011

[3] Dakuang Han: Discussions on concepts, countermeasures and technical routes for the redevelopment of high water-cut oilfields, Petroleum Exploration and Development Vol.37 No.5, 2010

[4] Ellen He, Shawn Han: Microrheology applications in the study of soft materials rheology, The $29^{\text {th }}$ Annual Conference of Chinese Chemical Society, 2014

[5] Zhang Rui, Wang Ruihe, Qiu Zhengsong, etc,: Stability evaluation of foam drilling fluid using light backscattering theory, Acta Petrolei Sinica Vol.26 No.1, 2005 